Parteienkritik und Parteiengeschichte: teilweise geglückter Start einer Buchreihe

Gonitzke, Andreas: „Innerparteiliche Demokratie“ in Deutschland. Das kritische Konzept und die Parteien im 20. Jahrhundert (DemOkrit-Reihe, Band 1), Martin Meidenbauer Verlagsbuchhandlung, München 2004, 184 Seiten, $€ 24,90$.

Nickel, Lutz: Dehler - Maier - Mende. Parteivorsitzende der FDP: Polarisierer - PräsidentGeneraldirektor (DemOkrit-Reihe, Band 2), Martin Meidenbauer Verlagsbuchhandlung, München 2005, 433 Seiten, $€ 49,90$.

Brauers, Christof: Die FDP in Hamburg 1945-1953. Start als bürgerliche Linkspartei. Mit einem Vorwort von Hildegard Hamm-Brücher (DemOkrit-Reihe, Band 3), Martin Meidenbauer Verlagsbuchhandlung, München 2007, 742 Seiten, € 39,90.

Es gibt eine neue Schriftenreihe: „DemOkrit - Studien zur Parteienkritik und Parteienhistorie“. Der Herausgeberkreis lässt verlauten, dass seine Gründung mit der verblichenen „Statt-Partei“ zu tun gehabt hätte. Diese ging am Widerspruch zwischen Anspruch und Wirklichkeit ein, „DemOkrit“ ist geblieben. Den Namen habe Winfried Steffani erfunden. Der Kreis orientiere sich an drei Wissenschaftlern: Heinrich von der Gablentz, der Parteien für unverzichtbar hielt; Gustav Radbruch, der die Parteien verfassungsrechtlich in Schach halten wollte und eben Winfried Steffani, für den Parteien „Interessenverbände in eigener Sache" waren. Einer der Herausgeber, Helmut Stubbe da Luz, entwirft im ersten Band der Reihe ein evolutionäres Menschheitsbild vom Häuptling bis hin zu Parteien in Zivilgesellschaften.

Als Eröffnung der Schriftenreihe folgt die Arbeit von Andreas Gonitzke, die dieser 2003 als Diplomarbeit an der Helmut-Schmidt-Universität der Bundeswehr in Hamburg eingereicht hatte. Seitdem ist der Autor Diplom-Politologe und Offizier im Bereich der operativen Information. Die Untersuchung von Lutz Nickel ist Band 2 der Schriftenreihe und wurde 2005 als Dissertation an der Georg-August-Universität Göttingen angenommen. Band 3, die Studie Christof Brauers', ist ebenfalls eine Dissertation, entstanden 2004 an der Helmut-Schmidt-Universität der Bundeswehr.

Die Arbeit von Andreas Gonitzke entspricht den Leitlinien der Herausgeber. Der Autor beschreibt das Konzept der innerparteilichen Demokratie als wichtiges Element des Staatsaufbaus. Es entstamme den Diskussionen der Weimarer Republik, habe dort jedoch keine Wirkung in der praktischen Politik entfaltet. Weil es der Weimarer Republik an „Demokraten, die willens gewesen wären, die verfassungsmäßige Ordnung mit allen Mitteln zu verteidigen", gemangelt habe (S. 69), habe die NSDAP als totalitäre Führerpartei aufsteigen und die Macht ergreifen können. Als Zeugen für die mangelnde innerparteiliche Demokratie schon zur Kaiserzeit zitiert Gonitzke vor allem Robert Michels und seine Oligarchiethese. Die Forderung nach innerparteilicher Demokratie stamme aus der Vorzeit der Bundesrepublik, die diesen Grundsatz in der Verfassung verankert hat. Der Autor zeichnet die entsprechenden Diskussionen im Parlamentarischen Rat und im Herrenchiemseer Verfassungskonvent nach. Im letzteren Gremium setzte sich der sozialdemokratische Jurist Hermann Louis Brill, von den Amerikanern eingesetzter und kurzzeitig amtierender Ministerpräsident und Innenminister von Thüringen, durch. Dieser forderte eine kurze und präzise Verankerung der wichtigsten Grundsätze der Parteiendemokratie in der Verfassung und 
einen Gesetzesauftrag. Dieses Gesetz sollte alsbald erlassen werden, und Brill formulierte hierfür Grundsätze innerparteilicher Demokratie, die niemals verwirklicht worden sind: jährliche Vorstandswahlen, Urabstimmungen über Wahlkandidaten, Begrenzung der Wahlkampfkosten oder Vorwahlen (S. 82 f.). Das Parteiengesetz kam erst 1967 - auf Druck des Bundesverfassungsgerichtes - zustande. Heute sind die von Brill geforderten Instrumente als Mittel zur Bekämpfung der Parteienverdrossenheit wieder aktuell. Obwohl das Parteiengesetz den vertikalen, funktionalen und regionalen Aufbau sowie die Grundrechte der Mitglieder der Parteien festlegt (S. 113 f.), erfüllt es nicht die strengen Demokratie-Kriterien Gonitzkes. Dazu zitiert er acht Autoren - unter anderem Wolfgang Abendroth, Karl Jaspers und Bodo Zeuner -, die alle, wenn auch von verschiedenen Wertepositionen aus, mehr innerparteiliche Demokratie forderten, als in Deutschland anzutreffen sei. Denen entgegen stellt der Autor Ulrich von Alemann, der auf nicht einmal einer Seite als „Beispiel für die apologetisierende Mehrheitsmeinung der deutschen Politologen“ erwähnt wird, die eine „relativ positive Bilanz“ zögen (S. 148). Das ist arg verkürzt und vereinfacht, so dass man den Wert der Arbeit von Gonitzke darin sehen kann, dass er die geringe innerparteiliche Demokratie in Deutschland zwar als ein Grundübel des Gemeinwesens herausstellt, den Mangel aber darin, dass der Autor den grundlegenden politikwissenschaftlichen Diskurs hierüber allenfalls rudimentär analysiert. So ist diese Publikation nur als Ergänzung zur betreffenden Literatur der „Apologeten“ zu empfehlen.

Im Gegensatz hierzu sind die Arbeiten von Lutz Nickel und Christof Brauers überaus material- und auch umfangreich.

Lutz Nickel unternimmt den Versuch, nicht nur die Biographie eines einzelnen Politikers zu analysieren; er untersucht gleich drei - alle nacheinander Parteivorsitzende der FDP. Die übergreifende Frage ist, wie es kommen konnte, dass die FDP nach den als problematisch zu beurteilenden Amtsperioden von Thomas Dehler und Reinhold Maier ab 1961 unter Erich Mende „als drittstärkste Kraft im Parteiensystem“ agieren konnte (S. 37). Bei seiner empirischen Analyse der Parteivorsitzenden - Thomas Dehler, der „Polarisierer“, Reinhold Maier, der "Präsident“ und Erich Mende, der "Generaldirektor“ - bemüht der Autor 14 Fragestellungen aus der Personalführungstheorie, wobei er die Rolle eines Parteivorsitzenden von der eines bloßen „Spitzenpolitikers“ abhebt. Im Sinne der Herausgeber der Reihe vergisst der Autor nicht, die Relevanz der innerparteilichen Demokratie herauszuheben, auf die es aber „in der harten Wirklichkeit“ nur noch selten ankäme (S. 26). Detailliert werden die politischen Aktivitäten der drei Vorsitzenden dargestellt, was zugleich eine Parteigeschichte aus der Vogelperspektive ergibt.

So ist die Rolle von Thomas Dehler beim „Aufstand der Jungtürken 1956“ interessant: Der Partei- und Fraktionsvorsitzende wehrte sich zwar gegen das Unions-Projekt eines "Grabenwahlsystems“ und ging auf der Bundesebene auf die SPD zu. Aber die Ablösung des CDU-Ministerpräsidenten Karl Arnold durch den Sozialdemokraten Fritz Steinhoff mittels eines von der FDP und ihren „Jungtürken“ mitgetragenen konstruktiven Misstrauensvotums in Düsseldorf erfüllte Thomas Dehler doch mit „einige(n) Sorgen“ über den Landesverband Nordrhein-Westfalen (S. 100). Schließlich konnte der Vorsitzende die Sezession wichtiger FDP-Politiker, die dann die - freilich erfolglose - „Freie Volkspartei (FVP)“ gründeten, nicht verhindern: Der Grundstein für seinen Abgang als Parteivorsitzender war gelegt.

Reinhold Maier, sein Nachfolger, ist in die FDP-Parteigeschichte als Befürworter des Konzeptes einer „liberalen Mitte“ (S. 165) eingegangen, das sich wegen seiner Milieube- 
schränkung nicht mit der Vorstellung der Jungtürken von der FDP als „dritter Kraft“ neben Union und SPD deckte. Nickel verdeutlicht, dass der Verzicht der FDP auf eine Koalitionsaussage bei der Bundestagswahl 1957 nicht Ausfluss des Konzeptes der liberalen Mitte war, sondern ein Kompromiss unterschiedlicher Strömungen in der Partei. Hier zeigte sich, dass Reinhold Maier kein wegweisender Parteiführer war, der Methode und Ziel vorgab.

Das war, der Analyse von Nickel folgend, auch Erich Mende nicht. Zwar erzielte dieser 1961 im Bundestagswahlkampf mit seinem Wahlkampfleiter Karl-Hermann Flach 12,8 Prozent und damit das beste Ergebnis der FDP überhaupt; zwar war er mit einer achtjährigen Amtszeit der bisher am längsten amtierende Vorsitzende der Freidemokraten, aber der Wahltriumph von 1961 verwandelte sich durch den „Umfall“ Erich Mendes in einen „Pyrrhussieg“ (S. 291): Der FDP-Vorsitzende war dem Taktiker Konrad Adenauer unterlegen. „Mit der CDU, aber ohne Adenauer“: Dieses FDP-Motto von 1961 artikulierte das Konzept vom liberalen Korrektiv, das zwar Wirksamkeit entfaltete, aber schon damals von der Parteiführung verdrängt wurde. Insofern ist Nickel zuzustimmen, wenn er schreibt: „Mende war kein Machtpolitiker“ - er sei „zu fair, taktvoll und verbindlich“ gewesen (S. 289). In gewisser Weise war er - was der Autor allerdings nicht schreibt - ein Illusionist und musste 1968 nach einem für ihn bitteren Parteitag den Stab an den Jungtürken Walter Scheel abgeben. Dieser sollte sich 1969 bei der Gründung der sozial-liberalen Koalition als wirklicher Parteiführer erweisen. Doch das ist nicht mehr das Thema der Arbeit von Nickel, die einerseits ein Stück Geschichte der FDP mit der Sicht auf die Vorsitzenden neu beleuchtet und andererseits den Versuch unternimmt, Elemente der Personalführungstheorie auf ihre Relevanz für die Spezies der Parteivorsitzenden hin zu untersuchen.

Neue Maßstäbe für historische und regionale Studien über politische Parteien setzt Christof Brauers schon quantitativ: Sein Buch hat mit Personenregister einen Umfang von 742 Seiten! Im Unterschied zu den reichlich vorhandenen Publikationen über die Geschichten einzelner FDP-Landesverbände, die fast stets offiziöse Selbstdarstellungen sind, ist diese Arbeit nicht nur quantitativ, sondern auch qualitativ außergewöhnlich, wissenschaftlich ambitioniert und nicht epigonenhaft.

Während die Untersuchung über die drei Parteivorsitzenden ein Stück FDP-Entwicklung aus der Vogelperspektive beschreibt, liefert Christof Brauers ein Bild aus der Froschperspektive über die Hamburger FDP. Diese hat lange Zeit innerparteilich durchaus erfolgreich gegen das aus Nordrhein-Westfalen, Niedersachsen und Hessen getragene Konzept der „nationalen Sammlung“ angekämpft. Dem diente zum Beispiel der Entwurf des linken „Liberalen Manifestes“ von 1952 als Gegenposition zu dem auf dem Emser Parteitag aus Nordrhein-Westfalen vorgelegten „Deutschen Programm“. Mit ihrem „Manifest“ konnten die Hamburger eine Festlegung der Bundespartei als Bürgerblock verhindern. Und in der nach dem ehemaligen NS-Staatssekretär Werner Naumann und seinen Unterwanderungsbestrebungen benannten „Naumann-Affäre“ von 1953 konnten die Hamburger innerparteiliche Untersuchungen und die Bloßstellung der Unterwanderer erreichen, so dass es auch ihr Erfolg war, dass das Konzept der nationalen Sammlung scheiterte (S. 696). Die Kraft zu solchem Einfluss zogen - wie Brauers darstellt - die Hamburger Liberalen aus ihrer demokratischen Vorgeschichte in der Weimarer Zeit und aus dem Widerstand jedenfalls vieler Linksliberaler gegen die „Machtergreifung“ der Nazis in der Hansestadt. Die linksliberalen Matadoren der sich nach 1945 stark in die Bundespolitik einbringenden FDP Hamburg hießen Hermann Schäfer, Willy Max Rademacher und Edgar Engelhard. So unterschiedlich diese Persönlichkeiten waren: An der Elbe wollten sie das alte Bündnis mit den Sozialde- 
mokraten aufleben lassen. Diese jedoch wähnten sich stark genug zum Alleinregieren, so dass der Autor zu einem der wichtigsten Ergebnisse über das Schicksal Linksliberaler in der FDP überhaupt kommt: „Was nützte Engelhard, Rademacher und der Parteilinken ihr sozialliberales Credo, die Propagierung eines ausgeglichenen Kurses der Mitte zwischen CDU und SPD, wenn die ,Liebe’ von der SPD nicht erwidert wurde.“ (S. 692) Dies scheint die Tragik der frühen Hamburger FDP zu sein.

Intensiv schildert Brauers die Vorgeschichte der Hamburger FDP in der Zeit von 1900 bis 1945. Auch unter den hanseatischen Linksliberalen gab es manche, die 1933 den Nazis als Alibi für ihre Machtergreifung dienten. Aber andere - und mehr als im übrigen Reich - wehrten sich schon früh gegen den anpasserischen Kurs der einst „linken“ DDP, die sich am Schluss „Staatspartei“ nannte und unterging. Der herausragendste unter ihnen war Hans Robinson, alteingesessener Textilkaufmann und Patron des liberalen Widerstandes an der Elbe gegen die NS-Diktatur. Ganz anders dagegen verhielt sich der Bürgermeister Wilhelm-Amsinck Burchard-Motz, der 1933 gleich zur NSDAP übertrat, 1934 aber gehen musste (S. 93).

Der Wert der Untersuchung über die FDP in ihrer Hamburger Frühzeit liegt darin, dass auch „Hamburger Spezialitäten“ vor dem Hintergrund der und in ihrer Bedeutung für die Bundespolitik analysiert werden. So wird ausführlich die Entstehung des „Hamburg Blocks“, der sich 1953 aus CDU, FDP und „Deutscher Partei“ (DP) bildete, dargestellt (S. 674). Das Buch von Brauers ist - alles in allem - eine Fundgrube für solche, die sich für die Zeitgeschichte Hamburgs, die Frühphase der FDP oder die Rolle der Liberalen in der Hansestadt oder für alle drei Themen und neue Erkenntnisse dazu interessieren.

Jürgen Dittberner

\section{Vertrauensbasis der Ostdeutschen: solide, aber zu knappe Studie}

Höhne, Benjamin: Vertrauen oder Misstrauen? Wie stehen die Ostdeutschen 15 Jahre nach der Wiedervereinigung zu ihrem politischen System?, Tectum Verlag, Marburg 2006, 155 Seiten, $€ 24,90$.

Dank der wohlgeordneten Gliederung des hier anzuzeigenden Bandes in Einleitung, Theorie, Analysekonzept, Empirische Überprüfung, Zusammenfassung und Ausblick fällt dem kundigen Leser bereits im Inhaltsverzeichnis auf, dass sich der Autor, Benjamin Höhne, nicht nur mit dem Vertrauen der Ostdeutschen in die Demokratie (diffuse Unterstützung), sondern auch mit ihrer Zufriedenheit (spezifische Unterstützung) auseinandersetzt und damit über die Ankündigung des Buchtitels hinaus nach den Grundlagen für die Stabilität der Demokratie in Ostdeutschland - einer zentralen Thematik der Politische-Kultur-Forschung - fragt (siehe auch S. 125). Das theoretische Fundament der Analysen ruht auf dem Konzept politischer Unterstützung David Eastons und auf Erklärungsansätzen für abweichende Werte des Institutionenvertrauens und der Demokratiezufriedenheit in Ostdeutschland (Situations- und Sozialisationshypothese). Leider fehlt zu beiden Hypothesen und bei der Abhandlung des Forschungsstandes die neueste Literatur (insbesondere Zeitschriftenartikel zum Thema) bis zum Abschlussjahr der Untersuchung 2004 (S. 60). 